\title{
Erratum to: Bloch-Wigner theorem over rings with many units
}

\author{
Behrooz Mirzaii
}

Published online: 18 July 2013

(C) Springer-Verlag Berlin Heidelberg 2013

\section{Erratum to: Math. Z. (2011) 268:329-346 \\ DOI 10.1007/s00209-010-0674-9}

\section{Introduction}

In this erratum, we correct a mistake that I made in [1]. The formulation of our main theorem [1, Theorem 5.1] is not correct. The correct form of the theorem, which is sufficient for our applications, is as follows:

Let $R$ be a commutative ring with many units and define

$$
\tilde{H}_{3}\left(\mathrm{SL}_{2}(R), \mathbb{Z}\right):=H_{3}\left(\mathrm{GL}_{2}(R), \mathbb{Z}\right) /\left(H_{3}\left(\mathrm{GL}_{1}(R), \mathbb{Z}\right)+R^{*} \cup H_{2}\left(\mathrm{GL}_{1}(R), \mathbb{Z}\right),\right.
$$

which is induced by the diagonal inclusion of $R^{*} \times \mathrm{GL}_{1}(R)$ in $\mathrm{GL}_{2}(R)$. Then, there is a quotient $M$ of $H_{1}\left(\Sigma_{2}, R^{*} \otimes R^{*}\right)$ which fits into exact sequences

$$
\begin{aligned}
0 \longrightarrow T_{R} \longrightarrow \tilde{H}_{3}\left(\mathrm{SL}_{2}(R), \mathbb{Z}\right) \longrightarrow \mathfrak{p}(R) \longrightarrow & \left(R^{*} \otimes_{\mathbb{Z}} R^{*}\right)_{\sigma} \longrightarrow K_{2}(R) \longrightarrow 0, \\
& \operatorname{Tor}_{1}^{\mathbb{Z}}(\mu(R), \mu(R)) \longrightarrow T_{R} \longrightarrow M \longrightarrow 0 .
\end{aligned}
$$

When $R$ is an integral domain, the left-hand side map in the second exact sequence is injective.

\section{The main theorem}

Lemma 3.1 in [1] is not correct. In fact, in the proof of the lemma, the map

$$
F_{q} \otimes \operatorname{Stab}_{\mathrm{GL}_{2}}(\infty) \mathbb{Z} \rightarrow F_{q} \otimes_{\mathrm{GL}_{2}} C_{1}\left(R^{2}\right), \quad s \otimes 1 \mapsto s \otimes(\infty, 0)
$$

The online version of the original article can be found under doi:10.1007/s00209-010-0674-9.

B. Mirzaii $(\varangle)$

Department of Mathematics, Institute for Advanced Studies in Basic Sciences,

P. O. Box 45195-1159, Zanjan, Iran

e-mail: bmirzaii@iasbs.ac.ir 
is not well defined. Because of this mistake, we made few uncorrect claims in [1]. Here, we give a correct formulations of the lemma and these claims that are sufficient for our applications. We follow the notations in [1].

Lemma 3.1 The groups $E_{1,0}^{2}$ and $E_{1,1}^{2}$ are trivial. Also, there is a surjective map $H_{1}\left(\Sigma_{2}, R^{*} \otimes\right.$ $\left.R^{*}\right) \rightarrow E_{1,2}^{2}$, where the action of $\Sigma_{2}=\{1, \sigma\}$ on $R^{*} \otimes R^{*}$ is defined by $\sigma(a \otimes b)=-b \otimes a$. In particular, $E_{1,2}^{2}$ is a 2-torsion group.

Proof In [1] on page 335, we have shown that $H_{0}\left(\mathrm{GL}_{2}, H_{1}(X)\right) \simeq \mathbb{Z}$ and $d_{2,0}^{1}=\mathrm{id}_{\mathbb{Z}}$. Consider the differential $d_{2,1}^{1}: E_{2,1}^{1}=H_{1}\left(\mathrm{GL}_{2}, H_{1}(X)\right) \rightarrow \operatorname{ker}\left(d_{1,1}^{1}\right) \simeq R^{*}$. Let $\varphi:$ $R^{*} \simeq H_{1}\left(\mathrm{GL}_{2}, C_{2}\left(R^{2}\right)\right) \rightarrow H_{1}\left(\mathrm{GL}_{2}, H_{1}(X)\right)$. It is easy to see that $d_{2,1}^{1} \circ \varphi=\mathrm{id}_{R^{*}}$. These facts immediately imply the triviality of $E_{1,0}^{2}$ and $E_{1,1}^{2}$. To compute $E_{1,2}^{2}$, first note that $\operatorname{ker}\left(d_{1,2}^{1}\right) \simeq H_{2}\left(R^{*}\right) \oplus\left(R^{*} \otimes R^{*}\right)^{\sigma}$. Again, one can easily see that the composition

$$
H_{2}\left(R^{*}\right) \simeq H_{2}\left(\mathrm{GL}_{2}, C_{1}\left(R^{2}\right)\right) \rightarrow H_{2}\left(\mathrm{GL}_{2}, H_{1}(X)\right) \rightarrow H_{2}\left(R^{*}\right) \oplus\left(R^{*} \otimes R^{*}\right)^{\sigma}
$$

is given by $x \mapsto(x, 0)$. Thus, $E_{1,2}^{2} \simeq\left(R^{*} \otimes R^{*}\right)^{\sigma} / A$. By an easy analysis of our main spectral sequence, we have

$$
E_{1,2}^{2}=\left(R^{*} \otimes R^{*}\right)^{\sigma} / A \hookrightarrow H_{3}\left(\mathrm{GL}_{2}\right) / H_{3}\left(R^{*} \times R^{*}\right) .
$$

We call this map $\beta$. We know that

$$
H_{1}\left(\Sigma_{2}, R^{*} \otimes R^{*}\right)=\frac{\left(R^{*} \otimes R^{*}\right)^{\sigma}}{(1+\sigma)\left(R^{*} \otimes R^{*}\right)}=\frac{\left(R^{*} \otimes R^{*}\right)^{\sigma}}{\left\langle a \otimes b-b \otimes a: a, b \in R^{*}\right\rangle} .
$$

Let $x_{a, b}=a \otimes b-b \otimes a$. Then,

$$
h=[(a, 1) \mid(1, b)]-[(1, b) \mid(a, 1)]-[(b, 1) \mid(1, a)]+[(1, a) \mid(b, 1)]
$$

is the cycle that represents the element $x_{a, b} \in\left(R^{*} \otimes R^{*}\right)^{\sigma} \subseteq H_{2}\left(R^{*} \times R^{*}\right)^{\sigma}$. Let $\tau$ be the automorphism of transposition of terms. Then, $\tau(h)-h=0$. Now, by [2, Lemma 2.5], the image of the class $\bar{h}$ under $\beta$ is given by $-\overline{\rho_{s}(h)}$, where $s=\left(\begin{array}{ll}0 & 1 \\ 1 & 0\end{array}\right)$ and

$$
\begin{aligned}
\rho_{s}(h):= & +[s|(1, a)|(b, 1)]-[(a, 1)|s|(b, 1)]+[(a, 1)|(1, b)| s] \\
& -[s|(b, 1)|(1, a)]+[(1, b)|s|(1, a)]-[(1, b)|(1,1)| s] \\
& -[s|(1, b)|(a, 1)]+[(b, 1)|s|(a, 1)]-[(b, 1)|(1, a)| s] \\
& +[s|(a, 1)|(1, b)]-[(1, a)|s|(1, b)]+[(1, a)|(b, 1)| s] .
\end{aligned}
$$

Now, by a direct computation, one can see that $\overline{\rho_{s}(h)}=0$. Thus, $x_{a, b} \in A$ and therefore $(1+\sigma)\left(R^{*} \otimes R^{*}\right) \subseteq A$. This implies the surjectivity that we are looking for.

Now, we are ready to correct Theorem 5.1 in [1].

Theorem 5.1 Let $R$ be a commutative ring with many units and define $\tilde{H}_{3}\left(\operatorname{SL}_{2}(R)\right):=$ $H_{3}\left(\mathrm{GL}_{2}\right) /\left(H_{3}\left(\mathrm{GL}_{1}\right)+R^{*} \cup H_{2}\left(\mathrm{GL}_{1}\right)\right)$. There is a quotient $M$ of $H_{1}\left(\Sigma_{2}, R^{*} \otimes R^{*}\right)$ which fits into exact sequences

$$
\begin{gathered}
0 \longrightarrow T_{R} \longrightarrow \tilde{H}_{3}\left(\mathrm{SL}_{2}(R)\right) \longrightarrow B(R) \longrightarrow 0 \\
\operatorname{Tor}_{1}^{\mathbb{Z}}(\mu(R), \mu(R)) \longrightarrow T_{R} \longrightarrow M \longrightarrow 0 .
\end{gathered}
$$

When $R$ is an integral domain, the left-hand side map in the second exact sequence is injective. 
Proof The proof is very similar to the proof of Theorem 5.1 in [1].

Remark 0.1 Here, we further make some minor corrections.

(i) In Proposition 2.1 and Remark 5.2 in [1], we have to assume that either the coefficient group is $\mathbb{Z}[1 / 2]$ or the ring $R$ has the property $R^{*}=R^{* 2}\left(K_{1}(R)=K_{1}(R)^{2}\right.$ for Remark 5.2).

(ii) The claim made in Remark 2.2 in [1] is not correct, and Suslin's claim in [2, Remark 2.2] remains true.

The rest of our claims in [1] remains true.

\section{References}

1. Mirzaii, B.: Bloch-Wigner theorem over rings with many units. Math. Z. 268, 329-346 (2011)

2. Suslin, A.A.: $K_{3}$ of a field and the Bloch group. Proc. Steklov Inst. Math. 183(4), 217-239 (1991) 